\title{
Phase-resolved Spectroscopy of the Crab Pulsar with XMM-Newton
}

\author{
M. G. F. Kirsch \\ European Space Agency (ESA), XMM-Newton Science Operations \\ Centre, Apartado - P.O. Box 50727, E-28080 Madrid, Spain \\ E. Kendziorra, R. Staubert \\ Institut für Astronomie und Astrophysik Universität Tübingen, \\ Abteilung Astronomie, Sand 1, D-72076 Tübingen, Germany
}

\begin{abstract}
The Crab pulsar (PSR B0531+21) has been observed with the EPIC-pn camera on board the ESA XMM-Newton satellite. The high time resolution of the EPIC camera $(7 \mu \mathrm{s})$ in its special Burst mode in combination with the huge effective area and the high quantum efficiency of the pn-CCD allows phase resolved spectroscopy of good quality over the energy range $0.6-8.5 \mathrm{keV}$. We performed phase resolved spectroscopy of the modulated flux of the Crab pulsar and found a significant hardening of the photon index in the inter-pulse region. Also the second pulse is harder in index by 0.1 than the first pulse.
\end{abstract}

\section{Introduction}

The first phase-resolved X-ray spectroscopy of the Crab pulsar was performed with OSO 8 (Pravdo \& Serlemitsos 1981). Analyzes with a finer phase resolution were published from $R X T E$ data (Pravdo, Angelini \& Harding 1997) and from BeppoSAX data (Massaro et al. 2000). Both measurements showed a significant hardening of the spectrum in the inter-peak region.

\section{Observations}

The Crab was observed with the EPIC-pn camera on board XMM-Newton in Burst mode in revolutions 234 (OBSID: 0135730701 with a total observation time of 10,000 sec) and 411 (OBSID: 0153750201, 0153750301 and 0153750301 with total observation times of $4634 \mathrm{~s}, 8583 \mathrm{~s}$ and $8583 \mathrm{~s}$ respectively). The three observations in revolution 411 were performed on a time basis of 30.3 h. The EPIC-pn camera, which provides high time resolution (Timing: $30 \mu \mathrm{s}$, Burst: $7 \mu \mathrm{s})$ and moderate energy resolution $(E / \Delta E=10-50)$ in the energy band $0.1-15 \mathrm{keV}$ is the ideal instrument to observe the Crab pulsar. In Burst mode the pile-up limit for a point source is $60,000 \mathrm{cts} / \mathrm{s}$, corresponding to a maximum flux of $6.3 \mathrm{Crab}$. The special read out in Burst mode leads to a loss of spatial resolution in the shift-direction. In Burst mode the lifetime is only $3 \%$, making it useful only for observations of very bright sources like the Crab. 
A more detailed description of the readout modes can be found in Kendziorra et al. (1999) and Kuster et al. (1999).

Table 1 summarizes the observations. The quoted lifetime also takes data losses due to telemetry constraints (counting mode) into account.

Table 1. Crab observations discussed in this paper.

\begin{tabular}{lcccc}
\hline OBS ID & rev. & $\begin{array}{c}\text { lifetime } \\
\text { [s] }\end{array}$ & mode+filter $^{a}$ & $\begin{array}{c}\text { pos. ang. } \\
\text { [deg] }\end{array}$ \\
\hline 0135730701 & 234 & 298.1 & BU T & 269.3 \\
0153750201 & 411 & 105.1 & BU M & 267.3 \\
0153750401 & 411 & 181.7 & BU M & 267.3 \\
0153750501 & 411 & 201.6 & BU M & 267.3 \\
\hline
\end{tabular}

${ }^{a}$ BU: Burst; T: Thick; M: Medium

\section{Data Analysis}

\subsection{Data reduction}

The data were processed with SAS 5.3.3. Event times were corrected to the solar system barycenter with the SAS tool barycen. For spectral analysis, only single events were used. Also (FLAG $=0$ ) was chosen to exclude border pixels for which the pattern type and the total energy is only known with lower precision. The Burst mode provides spatial resolution only in RAW-X direction (perpendicular to the CCD readout direction). We excluded events with (RAW$\mathrm{Y} \geq 142$ ) in order not to contaminate the spectra with pile-up events that could be generated in rows 142-180 during the readout in Burst mode.

\subsection{Epoch folding}

The best period for the Crab pulsar has been derived by using the epoch folding technique. The value for the period $P$ was derived for the observations in revolutions 234 and 411 separately by using as start values extrapolated data from the Jodrell Bank Crab pulsar monthly ephemeris (http://www.jb.man.ac.uk). The best X-ray periods were 33.52130947(1) ms for the epoch MJD 51988.640 (rev. 234) and 33.534100459(1) ms for the epoch MJD 52340.682 (rev. 411). Thus the relative deviation in the observed pulse period with respect to the most accurate radio data available $(\Delta P / P)$ was a few times $10^{-9}$. For joint analysis, the three observations in revolution 411 have been merged and with a phase shift adjusted to the epoch of the observation of revolution 234. After that all four observations of revolution 234 and 411 have been merged for further data analysis. 


\subsection{Localization of the pulsar}

The spatial resolution in Y-direction is lost in Burst mode. The position of the pulsar in the $\mathrm{X}$-direction was found by folding events in a running window of three columns with the best-fit period.

The flux for each of those windows was calculated using the OFF-pulse region (phase 0.8-1.0, see Fig. 1) as background. The strongest pulsed flux was found when the window was centered on column 37. To determine the pulsar spectrum we used a six column-wide window $\left(24^{\prime \prime}\right)$ centered on column 37 .

\subsection{Pulse phase spectroscopy}

Events from the pulsar region (columns 34-40) were sorted into seven phase intervals of the common pulse profile (see Fig.1). As background we used the OFF-pulse region with phase 0.8-1.0. For each of those phase intervals we computed the photon index with fixed $N_{\mathrm{H}}\left(2.68 \times 10^{21} \mathrm{~cm}^{-2}\right)$ derived from the OFF-pulse spectrum. The reduced $\chi^{2}$ values are generally acceptable within a range of 1.2-1.3. We see a change of the spectral index correlated with the pulse phase (see Fig. 1). The second pulse shows a harder spectrum than the first one and the intermediate pulse region again has a harder spectrum than the two pulses. The spectrum of the intermediate pulse region is harder than the spectrum of the main pulse by 0.3 . The spectrum of the second pulse is harder than the spectrum of the main pulse by 0.1 .

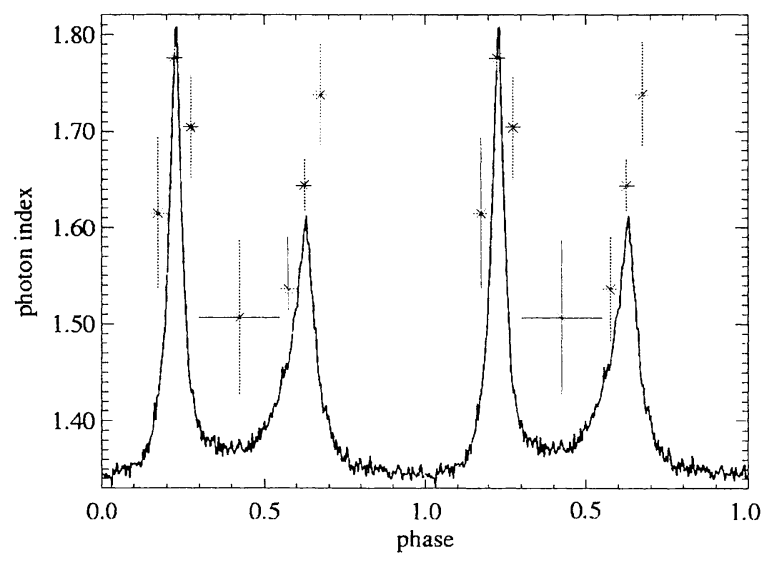

Figure 1. Pulse profile of the Crab pulsar from the three observations in rev. 411. 250 phase bins in the energy range from $0.6-15 \mathrm{keV}$ were used. For clarification the pulse profile is shown twice. Overlayed is the evolution of the photon index where the OFF-pulse region (phase 0.8-1.0) is used for background subtraction. Consequently, no photon index values can be determined in that region. 


\section{Discussion}

Our analysis gives qualitatively the same results as Massaro et al. (2000) and Pravdo et al. (1997) for the behavior of the photon index of the modulated flux of the Crab pulsar.

The analysis of Massaro et al. (2000) showed that a two-component emission model can account for the variation of the pulse profile with energy and the behavior of the spectral index with phase. However, no complete model for the $\mathrm{X}$-ray emission from the Crab pulsar was provided.

In three-dimensional outer magnetosphere models (Cheng, Ruderman \& Zhang 2000) the extension of the outer gap along both radial and azimuthal directions are limited by photon-photon pair production. Cheng et al. obtained the radial extension where pair production takes place. Later, Zhang \& Cheng (2002) presented a theoretical study of phase-dependent X-ray emission from the Crab pulsar. A three-dimensional outer-gap model is proposed, where the $\mathrm{X}$-ray emission is produced by synchrotron radiation of the secondary $e^{ \pm}$pairs, sensitive to the local properties of the emission regions. They calculated X-ray pulse profiles for different energy bands and spectral indices for different phases. The model reproduces approximately the basic properties of the results of the phase-resolved spectroscopy by Massaro et al. (2000) and our analysis.

Acknowledgments. The XMM-Newton project is an ESA Science Mission with instruments and contributions directly funded by ESA Member States and the USA (NASA). The German contribution of the XMM-Newton project is supported by the Bundesministerium für Bildung und Forschung/Deutsches Zentrum für Luft- und Raumfahrt. Many thanks to Andrea Tiengo and Markus Kuster who helped with the first steps of the data analysis and Uwe Lammers for processing the 234 data "mit der neuesten SAS Technologie".

\section{References}

Cheng, K. S., Ruderman, M., \& Zhang, L. 2000, ApJ, 537, 964

Kendziorra, E., Colli, M., Kuster, M., Staubert, R., Meidinger, N., \& Pfeffermann, E. 1999, in Proc. of the SPIE, EUV, X-Ray, and Gamma-Ray Instrumentation for Astronomy X, eds. O. Siegmund, \& K. A. Flaanagan, 3765 , p. 204

Kuster, M., Benlloch, S., Kendziorra, E., \& Briel, U. G. 1999, in Proc. of the SPIE, EUV, X-Ray, and Gamma-Ray Instrumentation for Astronomy X, eds. O. Siegmund, \& K. A. Flaanagan, 3765, p. 673

Massaro, E., Cusumano, G., Litterio, M., \& Mineo, T. 2000, A\&A, 361, 695

Pravdo, S. H., \& Serlemitsos, P. J. 1981, ApJ, 246, 484

Pravdo, S. H., Angelini, L., \& Harding, A. K. 1997, ApJ, 491, 808

Zhang, L., \& Cheng, K. S. 2002, ApJ, 569, 872 